\title{
Leadership and teaching in airway management
}

\author{
François Lemay, MD (1) • Mathieu Asselin, MD, FRCPC • Pascal Labrecque, MD, FRCPC
}

Received: 1 April 2021/Revised: 1 April 2021/Accepted: 1 April 2021/Published online: 6 July 2021

(C) Canadian Anesthesiologists' Society 2021

In this issue of the Journal, Howard et al. report on a survey on the use of guidelines for the management of the unanticipated difficult airway in Southwestern Ontario. ${ }^{1}$ Their results raise important questions and draw attention to organisational and educational factors related to airway emergencies preparedness.

Failed airways do happen in our country. The true incidence is uncertain as retrospective data do not account for "near miss" situations and potentially underreported complications. Closed claims analyses in Canada and the United States have shown that, although adverse events did occur among patients without obvious predictors for a difficult airway, pre-induction airway assessment was often incompletely recorded. ${ }^{2,3}$ In many cases, algorithms were not planned and front of neck airway access (FONA) was implemented too late. The large-scale $4^{\text {th }}$ National Audit Project (NAP4) had already drawn the same conclusions ten years ago in the United Kingdom (UK). ${ }^{4}$

There is still room for improvement in our capacity to prevent airway-related brain injury and death. Airway management is one of the few moments in anesthesia where our actions will directly impact the outcome. This is

F. Lemay, MD ( $\square)$

Département d'anesthésiologie, CHU de Québec - Université Laval, Hôtel-Dieu de Québec, 11 Côte du Palais, Quebec City, QC G1R 2J6, Canada

e-mail: francois.lemay.5@ulaval.ca

M. Asselin, MD, FRCPC

Département d'anesthésiologie, Hôpital Enfant-Jésus, CHU de

Québec - Université Laval, Quebec City, QC, Canada

P. Labrecque, MD, FRCPC

Département d'anesthésiologie et de soins intensifs, Université Laval, Hôtel-Dieu de Québec, Quebec City, QC, Canada one contributing factor to the stress imposed during an airway emergency. Having clear, pre-emptive, and proactive strategies for difficult airways is one measure to mitigate stress and maintain performance under such pressure. Various organizations such as the Canadian Airway Focus Group (CAFG), the American Society of Anesthesiologists (ASA) and the Difficult Airway Society (DAS) have published difficult airway algorithms to address this need. ${ }^{5-8}$ Guidelines from other organizations and/or addressing specific situations on the same topic have flourished in recent years. ${ }^{9}$ That may have contributed not only to the awareness of the necessity for an airway strategy but also to some confusion among airway practitioners. Howard et al. found that among consultant and trainee anesthesiologists of the London (Ontario) academic centre, the CAFG recommendations were the most popular. Yet, these did not reach the network of smaller community-affiliated hospitals where only the ASA guideline had been formally adopted.

Limited translation of clinical guidelines into practice is a known challenge highlighted by Howard et al. They suggest that, as opposed to the ASA and the DAS guidelines, that a lack of formal endorsement by a society contributed to the limited dissemination and adoption of the CAFG recommendations in Southwestern Ontario. We suspect that the same could be observed in the rest of Canada. The eventual endorsement of airway guidelines by a national organization such as the Canadian Anesthesiologists Society (CAS) would certainly weigh on their implementation among its members and other airway practitioners. The CAS has a definitive clout in anesthetic practice across our country. Over 45 years ago, in 1975, it adopted the very first Guidelines for Standards of Practice - the first guidelines by any anesthesia society. ${ }^{10}$ Today, through frequent updates, the CAS' Guidelines to 
the Practice of Anesthesia still support anesthesiologists in promoting and providing safe patient care in their own institutions. ${ }^{11}$ Airway guidelines certainly adhere to those same goals.

Howard et al. reported that only half of trainee anesthesiologists considered themselves adequately trained for difficult airways. This statement is difficult to interpret because, despite numerous options and tools mentioned in the National Curriculum Guide for Canadian Anesthesiology Residency from the Royal College of Physicians and Surgeons, ${ }^{12}$ there is no other consensus, guidance, or discrete definition of what constitutes readiness for difficult airways. Trainees must not only learn how to perform FONA and rescue tracheal intubation through extraglottic devices but also have the capacity to handle a comprehensive array of options and adjuncts to maintain oxygenation while establishing priorities and being able to deal with human factors, contextual issues, and ethics during an airway crisis. This cannot be achieved simply by attending training courses. Trainees must be given the opportunity to practice and demonstrate their skills. ${ }^{13}$ Simulation labs have substantially helped creating those opportunities and clinical airway rotations aim at enhancing those skills. The Entrustable Professional Activities for Anesthesiology recommends to collect observations from a large breadth of both anticipated and unanticipated difficult airway with the expectations not all will be achieved, with a minimum of six observations of achievement in a clinical setting. Those six observations do not confirm competency in awake intubation, for example, where the number required to achieve proficiency only with a flexible scope is considered more than three times higher. ${ }^{14}$ Crosby and Lane have thoroughly shown the challenges in airway training in Canada back in $2009 .{ }^{15}$ In the light of the results reported by Howard et al., many of the reflections and solutions Crosby and Lane had proposed continue to be relevant. This could serve as an opportunity for anesthesiologists involved in airway education to survey once again training across the country and discuss their airway curriculums. At our institution, we have recently revised our airway teaching program to ensure that essential airway techniques are taught to our residents. We have limited our references to recommendations to those from the CAFG and the DAS to ensure consistency. We have increased the accessibility to airway workshops to promote contextual learning and resource management training, while having a dedicated airway rotation and an otorhinolaryngology rotation. Residents from other programs such as Critical Care and Emergency Medicine have been included in our workshops. The Table lists, as an example, the airway workshops we offer to anesthesiology residents throughout their training. We still have to measure the long-term impact of this training on our graduates regarding their confidence in managing difficult airways.

Forty-three percent of respondents of the survey by Howard et al. never practiced FONA and only $13 \%$ did so within the last year. This potentially explains why only $18 \%$ of consultant and $7 \%$ of trainee anesthesiologists reported that they were confident with scalpel cricothyroidotomy. Emergency FONA is a rare event, happening only once in every 50,000 general anesthetics. ${ }^{4}$ Therefore, the ability to timely and adequately perform FONA is mostly gained through training. Frequent learning opportunities are required to promote skill mastery and long-term retention. ${ }^{16}$ This relies once again on hands-on workshops. The National Curriculum asks anesthesiologists to demonstrate a working knowledge of surgical options for emergency airway management. The anesthesiologist must demonstrate knowledge of the use of at least one cricothyrotomy kit or approach (minitracheostomy, cricothyrotomy, jet ventilation). FONA should be feasible in any location where airway management occurs. Added to the risks of jet ventilation in "can't intubate can't oxygenate" emergencies and the benefits of standardizing such a procedure, ${ }^{17}$ we have made the decision for our institution's airway curriculum to teach only the scalpel-bougie-tube technique promoted by the DAS. ${ }^{7}$ In our most recent survey on our FONA workshop, residents from Anesthesiology and Emergency Medicine provided positive comments on its structure and its perceived impact to improve skills in surgical cricothyrotomy. Narrative comments from the participants highlighted the importance of performing the procedure many times. Once again, we still have to measure the long-term impact of such training on residents; we intend to implement regular rehearsals of the FONA technique for consultants and operating room staff.

Finally, Howard et al. mention the fruitful implementation of an Airway Leads network in the UK following the publication of the NAP4. Airway Leads is endorsed by The Royal College of Anaesthetists and advocates for organizational strategies in airway management at local and national levels. ${ }^{18}$ The Australian and New Zealand College of Anaesthetists, through its Airway Management Special Interest Group, does so with its Airway Leads network. In Canada, other than the CAFG, there is no national airway interest group or formal Airway Leads network. We have not heard of any at the provincial level either. This is unfortunate, and the ongoing COVID-19 pandemic illustrates a concrete example of the importance of networking and collaboration in airway management. Particularly at the beginning of the pandemic, there was a need to share protocols regarding personal protective equipment for airway management in COVID-19 patients. Early 
information sharing was mostly done through personal communication and social media without dedicated channels. At the same time, COVID-19 airway management protocols were being developed through local organizations, which were not necessarily consistent with the recommendations eventually published by the CAS. ${ }^{19}$ Had it existed prior to the pandemic, a Canadian airway network could have promoted efficient leadership and expertise in airway management at both local and national levels during this time.

Anesthesiologists are considered airway experts by definition. Interestingly, in Canada, airway management has not been the specific focus of traditional interest groups such as those for regional and obstetric anesthesia, which, among others, are formerly hosted as distinct Sections within the framework of the CAS. Anesthesiologists must assume leadership in airway management organization and reach airway practitioners from other specialities. One step in this direction could be the networking of airway leaders in this country, formerly hosted within a section of the CAS which would welcome anesthesiologists with a particular interest in airway management. Such an Airway Management Section could nurture development, knowledge, and teaching of airway management strategy and techniques among its members. It could also link with other societies and the CAFG. This would contribute to and support the latter three-decadesold effort at promoting safe airway management in Canada.

\section{Prise en charge des voies aériennes : leadership et enseignement}

Dans ce numéro du Journal, Howard et coll. rapportent les résultats d'une enquête sur l'utilisation de lignes directrices pour la prise en charge des voies respiratoires difficiles non anticipées dans le Sud-Ouest de l'Ontario. ${ }^{1}$ Leurs résultats soulèvent d'importantes questions et attirent l'attention sur les facteurs organisationnels et éducatifs concernant le degré de préparation pour répondre aux urgences liées aux voies aériennes.

Les échecs d'intubation existent bel et bien au Canada. L'incidence réelle de cette complication est incertaine, car les données rétrospectives ne tiennent pas compte des situations de « quasi-échec » ('near miss'), ni des complications potentiellement sous-déclarées. Des analyses des réglements dans les poursuites médicales au Canada et aux États-Unis ont démontré que, bien que des événements indésirables se soient produits chez des patients qui ne présentaient pas de prédicteurs évidents de voies aériennes difficiles, l'évaluation des voies aériennes pré-induction était souvent enregistrée de manière incomplète. ${ }^{2,3}$ Dans de nombreux cas, les algorithmes n'avaient pas été planifiés et la cricothyroïdotomie a été mise en œuvre trop tard. Le $4^{\text {ème }}$ Projet d'audit national britannique (National Audit Project - NAP4) avait déjà tiré les mêmes conclusions il y a dix ans au Royaume-Uni. ${ }^{4}$

Nous pouvons encore faire des progrès dans notre capacité à prévenir les dommages cérébraux et décès liés à la prise en charge des voies aériennes. La prise en charge des voies aériennes est l'un des rares moments en anesthésie où nos actions ont un impact direct sur le devenir. Il s'agit d'un facteur contributif au stress imposé en cas d'urgence au niveau des voies aériennes. L'une des mesures pour atténuer ce stress et maintenir l'exécution sous une telle pression consiste à disposer de stratégies claires, préventives et proactives pour la prise en charge des voies aériennes difficiles. Divers organismes tels que le Canadian Airway Focus Group (CAFG), l'American Society of Anesthesiologists (ASA) et la Difficult Airway Society (DAS) ont publié des algorithmes concernant les voies aériennes difficiles pour répondre à ce besoin. ${ }^{5-8}$ Plusieurs lignes directrices d'autres organismes et/ou traitant de situations spécifiques sur le même sujet ont foisonné ces dernières années. ${ }^{9}$ Cela a peut-être contribué non seulement à la prise de conscience de la nécessité d'une stratégie pour la prise en charge des voies aériennes, mais aussi à une certaine confusion parmi les praticiens. Howard et coll. ont constaté que, parmi les anesthésiologistes et les résidents du centre universitaire de London (Ontario), les recommandations du CAFG étaient les plus populaires. Pourtant, ces lignes directrices n'ont pas percolé dans le réseau des petits hôpitaux communautaires affiliés, dans lesquels seules les recommandations de l'ASA ont été officiellement adoptées.

Le transfert limité des lignes directrices cliniques dans la pratique est un défi connu mis en évidence par Howard $e t$ coll. Ces auteurs suggèrent que, contrairement aux recommandations de l'ASA et de la DAS, le manque d'approbation officielle d'une société médicale a contribué à la diffusion et à l'adoption limitées des recommandations du CAFG dans le Sud-Ouest de l'Ontario. Nous soupçonnons qu'on pourrait observer la même chose ailleurs au Canada. Le soutien éventuel des lignes directrices concernant les voies aériennes par un organisme national comme la Société canadienne des anesthésiologistes (SCA) contribuerait certainement à leur application par ses membres et d'autres praticiens en charge des voies aériennes. La SCA dispose d'une influence indubitable sur la pratique de l'anesthésie au pays. Il y a plus de 45 ans, en 1975, elle adoptait le tout 
TABLE Airway workshops offered during the anesthesiology residency program at Université Laval

\begin{tabular}{|c|c|c|c|}
\hline Workshop & Participants & Duration & Content \\
\hline Basic airway management & PGY1 & $8 \mathrm{hr}$ & $\begin{array}{l}\text {-Airway evaluation } \\
\text { - Airway obstruction management } \\
\text { - Oxygenation devices } \\
\text { - Face-mask ventilation } \\
\text {-Direct laryngoscopy and videolaryngoscopy } \\
\text {-EGD } \\
\text { - Oral and nasal intubation. }\end{array}$ \\
\hline Advanced airway management & PGY2 & $8 \mathrm{hr}$ & $\begin{array}{l}\text {-Difficult airway algorithms (CAFG and DAS) } \\
\text {-Airway ultrasound } \\
\text {-Flexible bronchoscopy } \\
\text {-Intubation via an EGD } \\
\text {-Video intubating stylets }\end{array}$ \\
\hline Surgical airway & PGY2 and PGY4 & $4 \mathrm{hr}$ & $\begin{array}{l}\text {-FONA options } \\
\text {-Scalpel-bougie-tube technique (dry lab and cadaver) }\end{array}$ \\
\hline Extubation & PGY2 & $4 \mathrm{hr}$ & $\begin{array}{l}\text {-DAS extubation guidelines } \\
\text {-Tracheal tube exchange techniques (oral-oral; oral-nasal; simple lumen- } \\
\text { double lumen, double lumen, simple lumen) }\end{array}$ \\
\hline Awake intubation & PGY3 & $3 \mathrm{hr}$ & $\begin{array}{l}\text {-Sedation in awake intubation } \\
\text {-Methods of topicalization (dry lab) }\end{array}$ \\
\hline Immersive simulation & PGY3 & $8 \mathrm{hr}$ & $\begin{array}{l}\text { - Five cases focused on crisis management in airway scenarios, including } \\
\text { pediatric cases }\end{array}$ \\
\hline
\end{tabular}

CAFG = Canadian Airway Focus Group; DAS = Difficult Airway Society; EGD = extraglottic device; FONA = front of neck airway access; PGY $=$ postgraduate year.

premier Guide des normes d'exercice - les premières lignes directrices de toute société d'anesthésie. ${ }^{10}$ Aujourd'hui, grâce à des mises à jour fréquentes, le Guide d'exercice de l'anesthésie de la SCA aide encore les anesthésiologistes à promouvoir et à procurer des soins sécuritaires aux patients dans leurs propres établissements. ${ }^{11}$ Les lignes directrices portant sur les voies aériennes adhèrent certainement à ces mêmes objectifs.

Howard et coll. rapportent que seulement la moitié des résidents en anesthésiologie se considéraient adéquatement formés pour prendre en charge des voies aériennes difficiles. Une telle déclaration est difficile à interpréter parce que, malgré les nombreuses options et outils mentionnés dans le Programme national de résidence en anesthésiologie au Canada du Collège royal des médecins et chirurgiens, ${ }^{12}$ il n'existe pas d'autre consensus, orientation ou définition distincte de ce que signifie être préparé à prendre en charge des voies aériennes difficiles. Les résidents doivent non seulement apprendre à effectuer une cricothyrö̈dotomie et une intubation trachéale de sauvetage à l'aide de dispositifs extraglottiques, mais également avoir la capacité de gérer un éventail complet d'options et de compléments pour maintenir l'oxygénation tout en établissant des priorités et en étant en mesure de gérer les facteurs humains, les problématiques contextuelles et l'éthique pendant une situation critique touchant les voies aériennes. On ne peut acquérir ce type de compétences en assistant simplement à des cours de formation. Les résidents doivent avoir la possibilité de pratiquer et de démontrer leurs compétences. ${ }^{13}$ Les laboratoires de simulation ont grandement contribué à créer ces occasions et les stages cliniques sur les voies aériennes visent à améliorer ces compétences. Les Activités professionnelles confiables en anesthésiologie recommandent de recueillir des observations dans un large éventail de voies aériennes difficiles anticipées et non anticipées, sans s'attendre à ce qu'elles soient toutes réussies, avec un minimum de six observations de réalisations dans un cadre clinique. Ces six observations ne confirment pas la compétence en matière d'intubation éveillée, par exemple, où le nombre de tentatives réussies requis pour atteindre la compétence avec un fibroscope flexible seulement est au moins trois fois plus élevé. ${ }^{14}$ Déjà en 2009, Crosby et Lane faisaient un excellent état des lieux des défis de formation au Canada pour la prise en charge des voies aériennes. ${ }^{15}$ À la lumière des conclusions rapportées par Howard et coll., bon nombre des réflexions et des solutions alors proposées par Crosby et Lane continuent d'être pertinentes. Cela pourrait être l'occasion pour les anesthésiologistes impliqués dans la 
TABLEAU Ateliers sur les voies aériennes offerts dans le cadre du programme de résidence en anesthésiologie de l'Université Laval

\begin{tabular}{|c|c|c|c|}
\hline Atelier & Participants & Durée & Contenu \\
\hline $\begin{array}{l}\text { Prise en charge des voies } \\
\text { aériennes de base }\end{array}$ & $\mathrm{R} 1$ & $8 \mathrm{~h}$ & $\begin{array}{l}\text {-Évaluation des voies aériennes } \\
\text {-Prise en charge de l'obstruction des voies aériennes } \\
\text {-Équipement d'oxygénation } \\
\text {-Ventilation au masque } \\
\text {-Laryngoscopie directe et vidéolaryngoscopie } \\
\text {-DEG } \\
\text {-Intubation orale et nasale. }\end{array}$ \\
\hline $\begin{array}{l}\text { Prise en charge avancée des } \\
\text { voies aériennes }\end{array}$ & $\mathrm{R} 2$ & $8 \mathrm{~h}$ & $\begin{array}{l}\text {-Algorithmes pour les voies aériennes difficiles (CAFG et DAS) } \\
\text { •Échographie des voies aériennes } \\
\text {-Bronchoscopie flexible } \\
\text {-Intubation via un DEG } \\
\text {-Stylets d'intubation vidéo }\end{array}$ \\
\hline Voies aériennes chirurgicales & $\mathrm{R} 2$ et $\mathrm{R} 4$ & $4 \mathrm{~h}$ & $\begin{array}{l}\text {-Options de cricothyroïdotomie } \\
\text {-Technique scalpel-bougie-tube (laboratoire virtuel et modèles cadavériques) }\end{array}$ \\
\hline Extubation & $\mathrm{R} 2$ & $4 \mathrm{~h}$ & $\begin{array}{l}\text {-Lignes directrices d'extubation de la DAS } \\
\text {-Techniques d'échange des tubes trachéaux (oral-oral; oral-nasal; simple lumière-double } \\
\text { lumière, double lumière, simple lumière) }\end{array}$ \\
\hline Intubation éveillée & R3 & $3 \mathrm{~h}$ & $\begin{array}{l}\text {-Sédation pour l'intubation éveillée } \\
\text { •Méthodes de topicalisation (laboratoire virtuel) }\end{array}$ \\
\hline Laboratoire de simulation & R3 & $8 \mathrm{~h}$ & $\begin{array}{l}\text { - Cinq cas concentrés sur la gestion de crise dans des situations de voies aériennes, y } \\
\text { compris des cas pédiatriques }\end{array}$ \\
\hline
\end{tabular}

CAFG = Canadian Airway Focus Group DAS = Difficult Airway Society $;$ DEG = dispositif extraglottique; $\mathrm{R}=$ année de résidence.

formation touchant les voies aériennes d'examiner une fois de plus la formation à travers le pays et de discuter de leurs programmes d'études sur les voies aériennes. Dans notre établissement, nous avons récemment procédé à la révision de notre programme d'enseignement sur les voies aériennes afin de nous assurer que les techniques essentielles de prise en charge des voies aériennes sont enseignées à nos résidents. Nous avons limité nos références aux lignes directrices du CAFG et de la DAS afin de garantir une certaine cohérence. Nous avons amélioré l'accessibilité aux ateliers sur les voies aériennes afin de promouvoir les apprentissages contextuels et la formation en gestion des ressources, tout en ayant un stage dédié aux voies aériennes et un stage en otorhinolaryngologie. Les résidents d'autres programmes tels que les soins intensifs et la médecine d'urgence ont été inclus dans nos ateliers. Le tableau énumère, à titre d'exemple, les ateliers sur les voies aériennes que nous offrons aux résidents en anesthésiologie tout au long de leur formation. Il nous faut encore mesurer l'impact à long terme de cette formation sur nos diplômés quant à leur aisance pour prendre en charge des voies aériennes difficiles.

Quarante-trois pour cent des répondants au sondage de Howard et coll. n'ont jamais pratiqué de cricothyrö̈dotomie, et seuls $13 \%$ l'ont fait au cours de la dernière année. Cela explique potentiellement pourquoi seulement $18 \%$ des anesthésiologistes et $7 \%$ des résidents en anesthésiologie ont déclaré qu'ils se sentaient à l'aise de réaliser une cricothyroïdotomie au scalpel. La cricothyroïdotomie d'urgence est un événement rare, qui ne se produit qu'une fois sur 50000 anesthésies générales. ${ }^{4}$ Par conséquent, la capacité de réaliser en temps opportun et adéquatement une cricothyroïdotomie est principalement acquise au cours d'une formation. Afin de promouvoir la maîtrise des compétences et la rétention à long terme, il est nécessaire d'offrir des possibilités d'apprentissage fréquentes. Le Programme national demande aux anesthésiologistes de démontrer une connaissance pratique des options chirurgicales pour la prise en charge des voies aériennes en situation d'urgence. L'anesthésiologiste doit démontrer une connaissance de l'utilisation d'au moins une approche ou une trousse de cricothyroïdotomie (mini-trachéostomie, cricothyroïdotomie, jet-ventilation). La cricothyroïdotomie devrait être réalisable dans n'importe quel emplacement où il $\mathrm{y}$ a prise en charge des voies aériennes. Outre les risques de la jet-ventilation dans les situations d'urgence "impossible d'intuber, impossible d'oxygéner » et les avantages de la standardisation d'une telle intervention, ${ }^{17}$ nous avons pris la décision, en ce qui a trait au programme d'enseignement sur les voies aériennes 
de notre institution, d'enseigner uniquement la technique scalpel-bougie-tube promue par la DAS. ${ }^{7}$ Dans notre plus récent sondage portant sur notre atelier de cricothyroïdotomie, les résidents en anesthésiologie et en médecine d'urgence ont émis des commentaires positifs sur la structure et l'impact perçu de l'atelier pour améliorer leurs compétences en cricothyroïdotomie chirurgicale. Les commentaires narratifs des participants ont souligné l'importance de réaliser l'intervention à plusieurs reprises. Dans ce cas aussi, nous devrons mesurer l'impact à long terme d'une telle formation sur les résidents; nous avons l'intention de mettre en œuvre des mises à niveau régulières de la technique de cricothyroïdotomie pour les anesthésiologistes et le personnel de salle d'opération.

Enfin, Howard et coll. mentionnent la mise en œuvre réussie d'un réseau de responsables des voies aériennes baptisé Airway Leads au Royaume-Uni à la suite de la publication du NAP4. Ce réseau est appuyé par le Collège royal des anesthésistes et plaide en faveur de stratégies organisationnelles pour la prise en charge des voies aériennes aux niveaux local et national. ${ }^{18}$ Le Collège australien et néozélandais des anesthésistes, par l'intermédiaire de son groupe d'intérêt spécial de prise en charge des voies aériennes, le fait également avec son réseau Airway Leads. Au Canada, excepté le CAFG, il n'existe pas de groupe d'intérêt national sur les voies aériennes ni de réseau officiel de responsables des voies aériennes tel Airway Leads. Nous n'avons pas connaissance d'un tel réseau à l'échelle provinciale non plus. C'est malheureux, et la pandémie actuelle de COVID19 est un exemple concret de l'importance du réseautage et de la collaboration dans la prise en charge des voies aériennes. En particulier au début de la pandémie, il était nécessaire de partager les protocoles concernant les équipements de protection individuelle pour la prise en charge des voies aériennes des patients COVID-19. L'échange opportun d'informations s'est principalement fait par le biais de communications personnelles et de médias sociaux, sans canaux dédiés. Parallèlement, des protocoles de prise en charge des voies aériennes dans les cas de COVID-19 étaient en cours d'élaboration par l'entremise d'organisations locales, mais ces protocoles n'étaient pas nécessairement conformes aux recommandations finalement publiées par la SCA. ${ }^{19}$ S'il avait existé avant la pandémie, un réseau canadien dédié aux voies aériennes aurait pu promouvoir un leadership et une expertise efficaces dans la prise en charge des voies aériennes aux niveaux local et national pendant cette période.

Par définition, les anesthésiologistes sont considérés comme des experts en matière de voies aériennes. Fait intéressant, au Canada, la prise en charge des voies aériennes n'a jamais été au centre d'un groupe d'intérêt traditionnel, tel que ce fut le cas pour l'anesthésie régionale et obstétricale, qui, entre autres, étaient autrefois constitués en sections distinctes au sein de la SCA. Les anesthésiologistes doivent assumer le leadership dans l'organisation de la prise en charge des voies aériennes et rejoindre les praticiens d'autres spécialités qui sont aux prises avec les voies aériennes. Le réseautage des chefs de file canadiens en voies aériennes serait un pas dans cette direction; un tel réseau était autrefois hébergé au sein d'une section de la SCA, laquelle accueillait des anesthésiologistes s'intéressant particulièrement à la prise en charge des voies aériennes. Une telle section de prise en charge des voies aériennes pourrait favoriser le développement, les connaissances et l'enseignement de stratégies et de techniques de prise en charge des voies aériennes parmi ses membres. Elle pourrait également établir des liens avec d'autres sociétés et le CAFG. Cela contribuerait à la promotion et au soutien des efforts déployés depuis trente ans au Canada pour une prise en charge des voies aériennes sécuritaire.

Disclosures François Lemay and Mathieu Asselin participated in the elaboration of the third Canadian Airway Focus Group consensusbased recommendations for management of the difficult airway. Pascal Labrecque participated in the elaboration of the first Canadian Airway Focus Group recommendations for management of the unanticipated difficult airway.

Funding statement None.

Editorial Responsibility This submission was handled by Dr. Stephan K.W. Schwarz, Editor-in-Chief, Canadian Journal of Anesthesia.

Déclaration François Lemay et Mathieu Asselin ont participé à l'élaboration des troisièmes recommandations consensuelles du Canadian Airway Focus Group pour la prise en charge des voies aériennes difficiles. Pascal Labrecque a participé à l'élaboration des premières recommandations du Canadian Airway Focus Group pour la prise en charge des voies aériennes difficiles non anticipées.

Déclaration de financement Aucune.

Responsabilité éditoriale Cet éditorial a été traité par Stephan K.W. Schwarz, rédacteur en chef, Journal canadien d'anesthésie.

\section{References}

1. Howard M, Noppens R, Gonzalez N, Jones PM, Payne SM. Seven years on from the Canadian Airway Focus Group Difficult Airway Guidelines: an observational survey. Can J Anesth 2021. Doi: https://doi.org/10.1007/s12630-021-02056-5

2. Joffe AM, Aziz MF, Posner KL, et al. Management of difficult tracheal intubation: a closed claims analysis. Anesthesiology 2019; 131: 818-29. 
3. Crosby ET, Duggan LV, Finestone PJ, Liu R, De Gorter R, Calder LA. Anesthesiology airway-related medicolegal cases from the Canadian Medical Protection Association. Can J Anesth 2021; 68: 183-95.

4. Cook TM, Woodall N, Frerk C; Fourth National Audit Project. Major complications of airway management in the UK: results of the Fourth National Audit Project of the Royal College of Anaesthetists and the Difficult Airway Society. Part 1: anaesthesia. Br J Anaesth 2011; 106: 617-31.

5. Law JA, Broemling N, Cooper RM, et al. The difficult airway with recommendations for management - part 1 - difficult tracheal intubation encountered in an unconscious/induced patient. Can J Anesth 2013; 60: 1089-118.

6. Law JA, Broemling N, Cooper RM, et al. The difficult airway with recommendations for management - part 2 - the anticipated difficult airway. Can J Anesth 2013; 60: 1119-38.

7. Frerk C, Mitchell VS, McNarry AF, et al. Difficult Airway Society 2015 guidelines for management of unanticipated difficult intubation in adults. Br J Anaesth 2015; 115: 827-48.

8. Apfelbaum JL, Hagberg CA, Caplan RA, et al. Practice guidelines for management of the difficult airway: an updated report by the American Society of Anesthesiologists Task Force on Management of the Difficult Airway. Anesthesiology 2013; 118: 251-70.

9. Edelman DA, Perkins EJ, Brewster DJ. Difficult airway management algorithms: a directed review. Anaesthesia 2019; 74: $1175-85$.

10. Shephard DA. Guidelines to the practice of anaesthesia. In: Shephard DA: Watching Closely Those Who Sleep: A History of the Canadian Anaesthetists' Society 1943-1993, Chapter Five. Toronto: Canadian Anaesthetists' Society; 1993: 83-100.

11. Dobson G, Chow L, Filteau L, et al. Guidelines to the practice of anesthesia - revised edition 2021. Can J Anesth 2021; 68: 92-129.
12. Cummings $M$, Levine $M$, Pellerin H, Raazi M, Stewart J, Pierce $D$. National Curriculum for Canadian Anesthesiology Residency Third Edition. The Royal College of Physicians \& Surgeons of Canada; 2020.

13. Baker PA, Feinleib J, O'Sullivan EP. Is it time for airway management education to be mandatory? Br J Anaesth 2016; 117(Suppl 1): i13-6.

14. Smith JE, Jackson AP. Learning fibreoptic endoscopy. Nasotracheal or orotracheal intubations first? Anaesthesia 2000; 55: 1072-5.

15. Crosby E, Lane A. Innovations in anesthesia education: the development and implementation of a resident rotation for advanced airway management. Can J Anesth 2009; 56: 939-59.

16. Nicholls D, Sweet L, Muller A, Hyett J. Teaching psychomotor skills in the twenty-first century: revisiting and reviewing instructional approaches through the lens of contemporary literature. Med Teach 2016; 38: 1056-63.

17. Duggan LV, Ballantyne Scott B, Law JA, Morris IR, Murphy MF, Griesdale DE. Transtracheal jet ventilation in the 'can't intubate can't oxygenate' emergency: a systematic review. Br J Anaesth 2016; 117: i28-38.

18. McNarry AF, Cook TM, Baker PA, O'Sullivan EP. The Airway Lead: opportunities to improve institutional and personal preparedness for airway management. Br J Anaesth 2020; 125: e22-4.

19. Canadian Anesthesiologists' Society. COVID-19 Recommendations during Airway Manipulation. Available from URL: https://www.cas.ca/CASAssets/Documents/News/UpdatedMarch-25-COVID-19_CAS_Airway_Vsn_4.pdf (accessed April 2021).

Publisher's Note Springer Nature remains neutral with regard to jurisdictional claims in published maps and institutional affiliations. 